\title{
NOUVELLE
}

\section{L'ostéocrine, une nouvelle molécule de signalisation osseuse}

Pierre Moffatt notables sont l'indian hedgehog/patched, hormone parathyroïdienne (PTH)peptide apparenté à la PTH/récepteur de la PTH, le facteur de croissance fibroblastique (FGF)/récepteur FGFR3 et le peptide natriurétique de type C (CNP)/ récepteur à activité guanylyl cyclase de type B (GC-B). D'autres encore conti-
Hôpital Shriners pour enfants, 1529, avenue Cedar, Montréal (Québec), H3G IA6 Canada. pmoffatt@shriners.mcgill.ca

nuent d'être décelés, notamment l'ostéocrine, une protéine que notre groupe a découverte lors d'un criblage visant l'identification du sécrétome des cellules de l'os [1]. Le gène de l'ostéocrine a également été cloné à partir du muscle, et nommée muscline [2]. Le gène codant l'ostéocrine est exprimé presque exclu-

leurs récepteurs respectifs dont les plus

A

humain

souris

serpent

salamandre

poulet

humain

souris

serpent

salamandre

poulet

peptide signal MIDWRLASAHF ILAVTLTLWS SGKVLSVDVTTTEAFDSGVIDVOSTPTVREEKSATDLTAKLLLLDELV MLDWRLASTHF ILAMIVMLWGSGKAFSVDLASQEF---GTASLQSPPTAREEKSATELSAKLLRLDDLV

TASEEKSATDLVAKILLLNELV MLESRFLCARFLIAVGLIQWNAGRLLQAAAP ESSDSSRLLDTGSHSASSEERAATDLVAKLILIDELV MIQFQLVVVHLALVITLLQWHSSSVLLAEAAPEPLEPSAALGMAAHPTASEEKSASSLAAKLLLIDELV

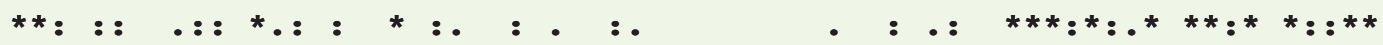
SLENDVIETKKKRSFSGFGSPIDRLSAGSVDHKGKORKVVDHPKRRFGIPMDRIGRNRLSNSRG-133 SLENDVFETKKKRSF SEFGSIDRLSAGSVEHRGKQRKAVDHSKKRFGIPMDRICRNRLSSSRG-130 SLENDVFETKKKRSFSGFGSP LDRLSVG---LKAKORKAVELPKKRFGIPLDRIGVNRLSGSRG SLENDVMETKKKRSFPGFGSPIDRLSAASTELKTKQRKVVEHPKRRFGVPLDRIGVNRLSNSRG-133 SLENEVTETKKKRSFPGEGSP IDRISATSVDAKGKQRKVVELPKRRFGVPLDRIGVSRIGNTKG-133

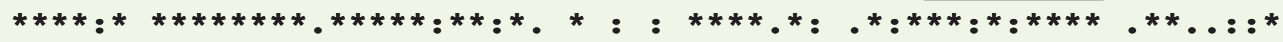

B

0stn-1

0stn-2

ANP

BNP

CNP
FöSPLDRLIS

FGIPMDRIG

SLRRSSCFGGRMDRIGAQSGLGCNSFRY

SPKMVQGSGCFGRKMDRISSSSGLGCKVLRRH GLSKGCFGLKLDRIGSMSGLGC
C

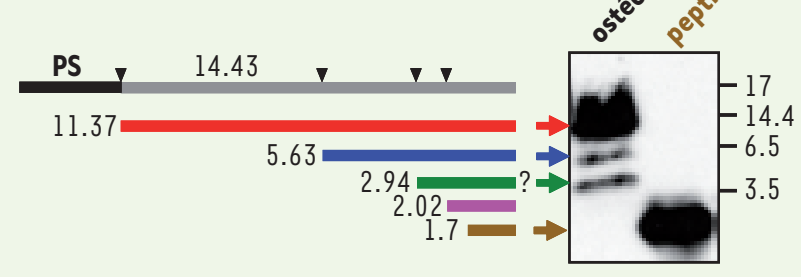

Figure 1. L'ostéocrine possède des motifs analogues aux peptides natriurétiques. A. Alignement de l'ostéocrine de différentes espèces et principales caractéristiques structurales. L’homologie des séquences est indiquées sous l'alignement (résidus identiques [*], conservés [:], et faiblement similaires [.]). La région du peptide signal est indiquée avec son site de clivage $(\downarrow)$. Les sites dibasiques sont surlignés en jaune et leurs sites de coupures potentiels sont indiqués $(\downarrow)$. B. Deux motifs présents dans l'ostéocrine (0stn- 1 et 0 stn-2) ont une séquence similaire aux peptides natriurétiques. Les acides aminés identifiés comme étant importants pour la liaison au NPR-C $(\bullet)$ sont conservés. C. L'analyse Western de l'ostéocrine sécrétée par les cellules HعK293 indique la présence d'un clivage. Les fragments anticipés avec leur taille respective (kDa) sont illustrés à gauche et, au moins, celui correspondant au premier site de coupure dibasique (bleu) a été confirmé par micro séquençage. 
sivement par les ostéoblastes [1] et les cellules musculaires [2-4]. L'ostéocrine est une protéine hautement conservée entre les espèces (Figure l) et l'examen de sa structure primaire met en évidence des motifs rappelant ceux entrant dans la composition de plusieurs prohormones [5]. En effet, nous avons démontré par immunobuvardage Western (Western blot) qu'il est possible que des fragments d'ostéocrine soient produits sous l'action de protéases qui reconnaissent des motifs à caractères dibasiques. Par contre, l'existence de ces fragments in vivo reste encore à établir, ainsi que la nature exacte de la protéase ou des protéases qui participent à ce processus. II est intéressant de penser que les clivages qui en résultent puissent libérer les parties actives de la molécule. Deux motifs ayant une similarité avec la famille des peptides natriurétiques (PN) sont bordés par ces sites de coupures potentiels (Figure 1 ). À l'instar des autres PN, l'absence de résidus cystéine dans l'ostéocrine indique que les fragments agiraient comme peptides linéaires. Les $P N$ et, particulièrement I'ANP (atrial natriuretic peptide) et le BNP (brain natriuretic peptide), constituent d'importants régulateurs de l'homéos- tasie du tonus vasculaire par leur action natriurétique et cardiaque [6].

II a été établi, en revanche, que le CNP exerce un rôle de régulateur local important particulièrement pour la croissance longitudinale des os longs $[7,8]$. Ainsi chez la souris l'inactivation du gène CNP ou l'inactivation de son récepteur GC$B$, doté d'une activité guanylyl cylase, conduit à un phénotype de nanisme. À l'inverse, l'inactivation du récepteur de clairance des PN (NPR-C) ou la surexpression du CNP entraînent une croissance exagérée des os longs chez la souris. Chez les mammifères, les trois composantes du système CNP/GC-B/NPR-C sont non seulement exprimées au niveau de la plaque de croissance, mais aussi dans les ostéoblastes. Cependant, les phénotypes observés dans les modèles animaux affectés de perturbations dans l'une ou l'autre des composantes CNP/GC-B/ NPR-C ont principalement été associés à un effet sur la plaque de croissance et non pas sur les ostéoblastes, où les trois composantes sont aussi présentes. Le système CNP/GC-B/NPR-C est aussi fonctionnel chez l'homme comme en témoignent des mutations décelées dans le gène du récepteur GC-B qui causent une forme de nanisme rare (type

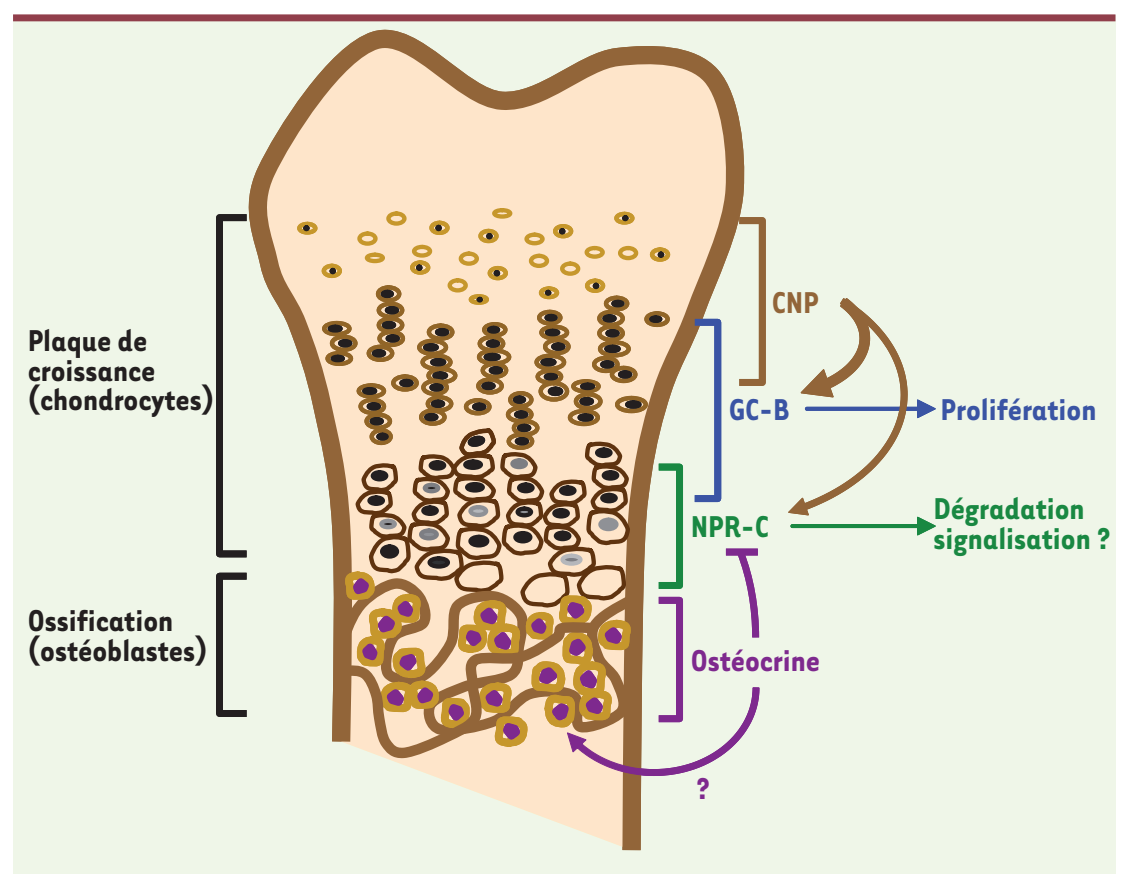

Maroteaux) dans laquelle la longueur des avant-bras, des mains et des jambes est principalement affectée [9]. Ces données illustrent l'importance fondamentale du système CNP/GC-B/NPR-C dans la physiologie osseuse et soulève la possibilité qu'il puisse être modulé par l'ostéocrine, étant donné ses caractéristiques structurales ressemblant aux PN.

\section{L'ostéocrine, un nouveau} ligand naturel du NPR-C

Jusqu'à tout récemment, le rôle joué par l'ostéocrine in vivo est demeuré largement inexploré. Pour mieux comprendre ce rôle, notre groupe a mis au point une souris transgénique chez laquelle l'ostéocrine est surexprimée dans les ostéoblastes [4]. Le phénotype observé, qui rappelle celui des modèles génétiquement modifiés du couple CNP/ GC-B/NPR-C, manifeste un accroissement significatif de la croissance des os longs, associé avec une légère augmentation de la prolifération des chondrocytes, ainsi qu'une gibbosité vertébrale. Pour éclaircir le mécanisme moléculaire d'action de l'ostéocrine et l'éventualité de son interaction avec le CNP/GC-B/ NPR-C dans l'os, nous avons réalisé une série d'études in vitro démontrant que l'ostéocrine se lie de manière spécifique avec le NPR-C mais pas avec le GC-A ni le GC-B. Cette liaison qui a été observée aussi bien avec la protéine entière qu'avec des peptides synthétiques, a une constante de liaison estimée à $5 \mathrm{nM}$. Le NPR-C étant considéré principalement comme un récepteur de clairance pour les PN, le mécanisme le plus plausible

Figure 2. Modèle d'action de l'ostéocrine dans les os longs en formation. L'ostéocrine secrétée par les ostéoblastes diffuserait dans la plaque de croissance ou elle se lierait au NPR-C. Cette liaison empêcherait celle du CNP et favoriserait l'activation du récepteur GC-B. Les domaines d'expression putatifs pour les différents facteurs sont indiqués. L'importance sur les ostéoblastes de la signalisation par l'ostéocrine est encore incertaine. 
envisagé consiste à considérer que l'ostéocrine empêche la dégradation du CNP et, indirectement, entraîne une activation de son récepteur GC-B (Figure 2). Nous avons validé cette possibilité en intensifiant la signalisation du CNP via son récepteur GC-B en présence d'ostéocrine dans des cellules chondrocytaires et ostéoblastiques qui expriment le GC-B et le NPR-C. En accord avec nos études in vitro, les niveaux mesurés du second messager GMP cyclique suscité par le $\mathrm{GC}-\mathrm{B}$, sont doublés dans les os longs des souris transgéniques qui surexpriment l'ostéocrine. L'ostéocrine sécrétée par les ostéoblastes adjacents aux plaques de croissance pourrait vraisemblablement se diffuser dans ces plaques et agir au niveau du NPR-C situé sur les chondrocytes hypertrophiés (Figure 2). Bien que la déficience en ostéocrine n'ait pas encore été clairement reliée à une maladie osseuse particulière, une approche génomique a établi une association significative entre un locus comprenant le gène ostéocrine et la longueur des os chez le poulet [10].

\section{Utilisation thérapeutique et perspectives futures}

Il est tentant de spéculer sur les applications thérapeutiques potentielles de l'ostéocrine dans le traitement du nanisme. Il a en effet été démontré qu'il existe une interaction entre les voies de signalisation du CNP/GC-B et du FGF/FGFR3 dans la plaque de croissance [11]. Ainsi, la surexpression de CNP dans les chondrocytes permet de normaliser la croissance des souris ayant une mutation activatrice du récepteur FGFR3. II est conceptuellement possible que l'ostéocrine puisse également corriger indirectement les anomalies de croissance chez ces mêmes souris, en empêchant la dégradation du CNP. Le croisement de souris transgéniques surexprimant l'ostéocrine avec de tels modèles de chondrodysplasie pourrait permettre de vérifier cette hypothèse. Plusieurs questions cependant demeurent encore sans réponse. Par exemple, quel est le rôle de l'ostéocrine dans le muscle et le mécanisme identifié dans l'os y est-il également opérationnel ? S'il existe des voies de communication entre les muscles, les tendons, les ligaments et l'os, l'ostéocrine y joue-t-elle un rôle de médiateur? Étant donné sa nature soluble, l'ostéocrine se retrouve-t-elle dans la circulation et pourrait-elle agir sur d'autres organes à distance, se qualifiant ainsi comme hormone? À l'aide d'autres modèles animaux, tels des knock-out spécifiques de l'ostéocrine dans l'os ou le muscle, il sera peut-être possible de répondre à certaines de ces interrogations. $\diamond$
Osteocrin is a new signaling

molecule acting on bone

\section{RÉFÉRENCES}

1. Thomas G, Moffatt P, Salois P, et al. Osteocrin, a nove bone-specific secreted protein that modulates the osteoblast phenotype. J Biol Chem 2003; 278: 50563-71.

2. Nishizawa H, Matsuda M, Yamada Y, et al. Musclin, a novel skeletal muscle-derived secretory factor. J Biol Chem 2004 ; 279 : 19391-5.

3. Banzet S, Koulmann N, Sanchez H, et al. Musclin gene expression is strongly related to fast-glycolytic phenotype. Biochem Biophys Res Commun 2007 ; 353: 713-8.

4. Moffatt P, Thomas G, Sellin K, et al. Osteocrin is a specific ligand of the natriuretic peptide clearance receptor that modulates bone growth. J Biol Chem 2007 ; 282 : 36454-62.

5. Marcinkiewicz M, Seidah N, Chretien M. Les convertases des prohormones et le système nerveux. Med Sci (Paris) $1993 ; 9: 553-61$.

6. Potter LR, Abbey-Hosch S, Dickey DM. Natriuretic peptides, their receptors, and cyclic guanosine monophosphate-dependent signaling functions. Endocrinol Rev 2006 ; 27 : 47-72.

7. Pejchalova K, Krejci P, Wilcox WR. C-natriuretic peptide : an important regulator of cartilage. Mol Genet Metab 2007 ; $92: 210$-5.

8. Olney RC. C-type natriuretic peptide in growth : a new paradigm. Growth Horm IGF Res 2006; 16 (suppl A) : S6-14.

9. Bartels CF, Bukulmez H, Padayatti P, et al. Mutations in the transmembrane natriuretic peptide receptor NPR$B$ impair skeletal growth and cause acromesomelic dysplasia, type maroteaux. Am J Hum Genet 2004; $75: 27-34$.

10. Zhou H, Deeb N, Evock-Clover CM, et al. Genome-wide linkage analysis to identify chromosomal regions affecting phenotypic traits in the chicken. III. Skeletal integrity. Poult Sci $2007 ; 86: 255-66$.

11. Yasoda A, Komatsu Y, Chusho H, et al. Overexpression of CNP in chondrocytes rescues achondroplasia through a MAPK-dependent pathway. Nat Med 2004 ; $10: 80-6$.

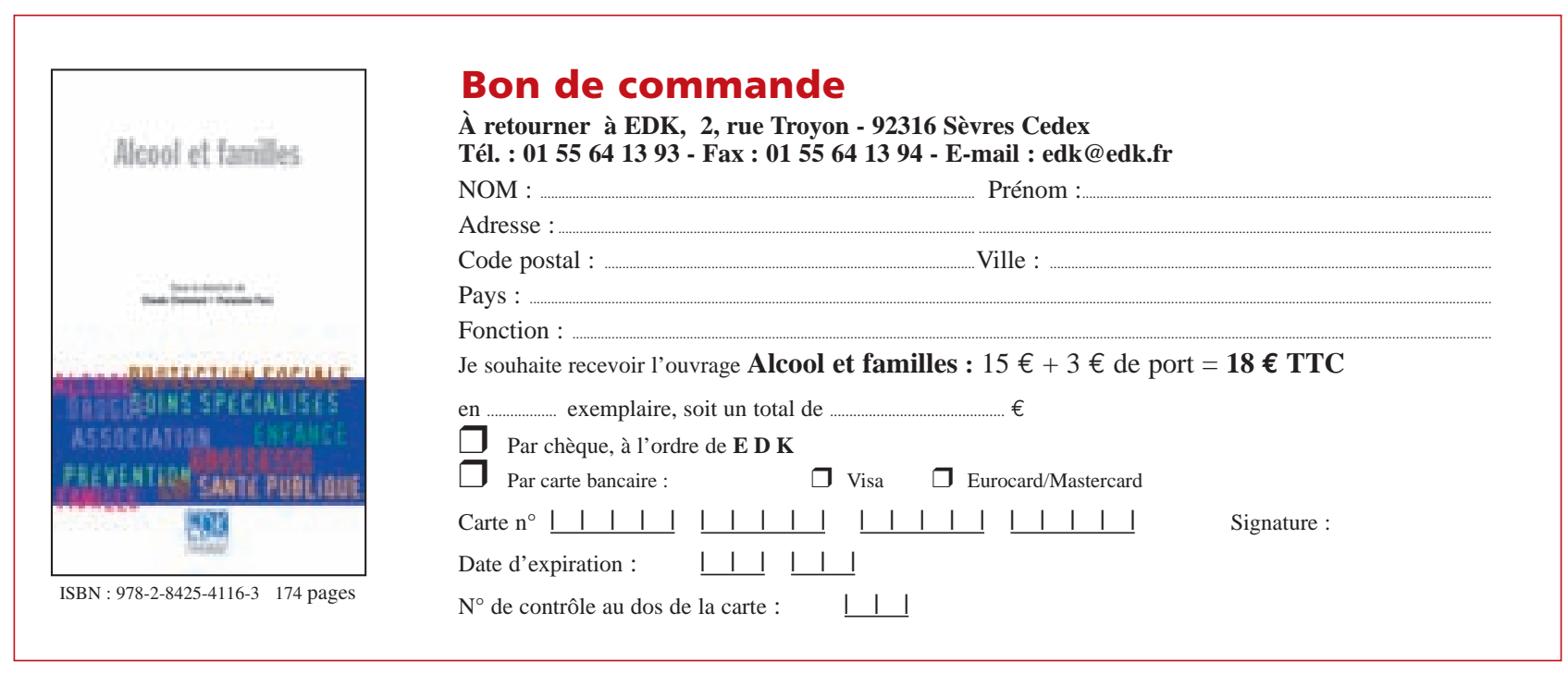

